\title{
The Family of Global Attractors for a Generalized Kirchhoff Equations
}

\author{
Guoguang Lin, Xiaomei Liu \\ Department of Mathematics, Yunnan University, Kunming, China \\ Email: gglin@ynu.edu.cn,790612351@qq.com
}

How to cite this paper: Lin, G.G. and Liu, X.M. (2021) The Family of Global Attractors for a Generalized Kirchhoff Equations. Open Journal of Applied Sciences, 11, 789-806.

https://doi.org/10.4236/ojapps.2021.117058

Received: June 1, 2021

Accepted: July 17, 2021

Published: July 20, 2021

Copyright $\odot 2021$ by author(s) and Scientific Research Publishing Inc. This work is licensed under the Creative Commons Attribution International License (CC BY 4.0).

http://creativecommons.org/licenses/by/4.0/

(c) (i) Open Access

\begin{abstract}
This paper deals with the existence and uniqueness of solutions of generalized Kirchhoff equations and the family of global attractors for the equation and its dimension estimation. First, the stress term $M\left(\left\|\nabla^{m} u\right\|_{p}^{p}\right)$ of Kirchhoff equation is properly assumed. When certain conditions are met between the order $m$ and the degree $p$ of Banach space $L^{p}(\Omega)$, the existence and uniqueness of the solution of equation are obtained by a prior estimation and Galerkin's method; Then, the bounded absorption set $B_{0 k}$ is obtained by prior estimation, and it is proved that the solution semigroup $S(t)$ generated by the equation has a family of global attractors $A_{k}$ in phase space $E_{k}=\left(H^{2 m+k}(\Omega) \cap H_{0}^{1}(\Omega)\right) \times H_{0}^{k}(\Omega)$ by using Rellich-Kondrachov compact embedding theorem. Further, the equation is linearized and rewritten into a first-order variational equation, and it is proved that the solution semigroup $S(t)$ is Fréchet differentiable on $E_{k}$; Finally, the upper bound of Hausdorff dimension and Fractal dimension of $A_{k}$ is estimated, and the Hausdorff dimension and Fractal dimension are finite.
\end{abstract}

\section{Keywords}

Kirchhoff Equation, Galerkin's Method, Family of Global Attractor,

Dimension Estimation

\section{Introduction}

This paper will study the initial-boundary value problems of the following generalized Kirchhoff equations:

$$
u_{t t}+M\left(\left\|\nabla^{m} u\right\|_{p}^{p}\right)(-\Delta)^{2 m} u+\beta(-\Delta)^{2 m} u_{t}+|u|^{\rho}\left(u_{t}+u\right)=f(x),
$$




$$
\begin{gathered}
u(x, t)=0, \frac{\partial^{i} u}{\partial v^{i}}=0, i=1,2, \cdots, 2 m-1, x \in \partial \Omega, t>0, \\
u(x, 0)=u_{0}(x), u_{t}(x, 0)=u_{t}(x), x \in \Omega \subset R^{n} .
\end{gathered}
$$

where $m \in N^{+}, \Omega \subset R^{n}(n \geq 1)$ is a bounded domain with smooth boundary $\partial \Omega, f(x)$ is an external force term, $M\left(\left\|\nabla^{m} u\right\|_{p}^{p}\right)$ is the stress term of Kirchhoff equation, $\beta>0, \beta\left(-\Delta^{2 m}\right) u_{t}$ is a strong dissipative term, $|u|^{\rho}\left(u_{t}+u\right)$ is a nonlinear source term.

Many scholars have studied the existence of global attractor of Kirchhoff equation with strong dissipative term, [1]-[7] can be referred.

In reference [8], scholars considered the following Kirchhoff type wave equation with nonlinear strong damping term

$$
\begin{gathered}
u_{t t}-\varepsilon_{1} \Delta u_{t}+\alpha\left|u_{t}\right|^{p-1} u_{t}+\beta|u|^{q-1} u-\varphi\left(\|\left.\nabla u\right|^{2}\right) \Delta u=f(x),(x, t) \in \Omega \times R^{+} \\
u(x, 0)=u_{0}(x) ; u_{t}(x, 0)=u_{1}(x), x \in \Omega \\
\left.u(x, t)\right|_{\partial \Omega}=0,\left.\Delta u(x, t)\right|_{\partial \Omega}=0, x \in \Omega
\end{gathered}
$$

Here, $\Omega$ is a bounded domain with smooth boundary $\partial \Omega$ in $R^{N}$, $\varepsilon_{1}, \alpha, \beta$ are normal numbers.

TokioMatsuyama and Ryolkehata [9] proved the global solution and attenuation of the solution of Kirchhoff type wave equation with nonlinear damping:

$$
u_{t t}-M\left(\|\nabla u(t)\|_{2}^{2}\right) \Delta u+\delta\left|u_{t}\right|^{p-1} u_{t}=\mu|u|^{q-1} u .
$$

With compact boundary conditions

$$
\left.u(x, t)\right|_{\partial \Omega}=0, t \geq 0 .
$$

FucaiLi discussed the higher-order Kirchhoff type equations with nonlinear terms in reference [10]:

$$
\begin{gathered}
u_{t t}+\left(\int_{\Omega}\left|\nabla^{m} u\right|^{2}\right)^{q}(-\Delta)^{m} u+u_{t}\left|u_{t}\right|^{r}=|u|^{p} u, x \in \partial \Omega, t>0, \\
u(x, t)=0, \frac{\partial^{i} u}{\partial v^{i}}=0, i=1,2, \cdots, m-1, x \in \partial \Omega, t>0, \\
u(x, 0)=u_{0}, u_{t}=u_{1}(x), x \in \Omega .
\end{gathered}
$$

In a bounded domain, where $m>1$ is a positive integer, $p, q, r>0$ is a normal number, if $p \leq r$, the existence of global solution will be obtained, if $p>\max \{r, 2 q\}$, for any initial value with negative initial energy, the solution explodes in a finite time. For more related research results, please refer to references [11] [12] [13] [14].

In this paper, for the convenience of narration, the following spaces and marks are defined: $H=L^{2}(\Omega), H_{0}^{2 m}(\Omega)=H^{2 m}(\Omega) \cap H_{0}^{1}(\Omega)$, $H_{0}^{2 m+k}(\Omega)=H^{2 m+k}(\Omega) \cap H_{0}^{1}(\Omega), \quad E_{k}=H_{0}^{2 m+k}(\Omega) \times H_{0}^{k}(\Omega)$, $(k=0,1,2, \cdots, 2 m)$. Define $(\cdot, \cdot)$ and $\|\cdot\|$ to represent the inner product and 
norm of $H$ respectively, namely

$$
(u, v)=\int_{\Omega} u(x) v(x) \mathrm{d} x,(u, u)=\|u\|^{2} .
$$

Let $A_{k}$ be the family of global attractor from $E_{0}$ to $E_{k}, B_{0 k}$ be the bounded absorption set in $E_{k}$, where $k=1,2, \cdots, 2 m . \quad C_{i}(i=0,1,2, \cdots)$ represents a constant.

(H1) assume that Kirchhoff type stress term $M(s) \in C^{2}([0,+\infty], R)$ satisfies:

$$
1<\mu_{0} \leq M(s) \leq \mu_{1}, \mu=\left\{\begin{array}{l}
\mu_{0}, \frac{\mathrm{d}}{\mathrm{d} t}\left\|\nabla^{2 m} u\right\|^{2} \geq 0, \\
\mu_{1}, \frac{\mathrm{d}}{\mathrm{d} t}\left\|\nabla^{2 m} u\right\|^{2}<0 .
\end{array}\right.
$$

where $\mu$ is a constant.

(H2) $\rho \leq \frac{8 m}{n}$

There exist constant $\varepsilon, 0<\varepsilon<\min \left\{\sqrt{1+\frac{\beta \lambda_{1}^{2 m}}{2}}-1,2 \mu_{0}, \frac{2 \mu_{0}}{\frac{1}{\lambda_{1}^{2 m}}+\beta}\right\}$.

\section{The Existence and Uniqueness of Global Solution}

In this section, under the assumption of Kirchhoff stress term, the existence and uniqueness of global solution are obtained by prior estimation and Galerkin's method.

Lemma 2.1 Suppose Kirchhoff stress term $M(s)$ satisfies the conditions (H1), Assume that (H2) holds, $f \in H,\left(u_{0}, v_{0}\right) \in E_{0}, v=u_{t}+\varepsilon u, \varepsilon>0$, then the smooth solution of the initial-boundary value problem (1.1)-(1.3) satisfies $(u, v) \in E_{0}, \quad u \in L^{\infty}\left(0,+\infty ; H^{2 m}(\Omega)\right), \quad v \in L^{\infty}\left(0,+\infty ; L^{2}(\Omega)\right) \cap L^{2}\left(0, T ; H^{2 m}(\Omega)\right)$, and satisfy that following inequality

$$
\begin{gathered}
\|(u, v)\|_{E_{0}}^{2}=\left\|\nabla^{2 m} u\right\|^{2}+\|v\|^{2} \\
\leq\left(\left\|v_{0}\right\|^{2}+\varepsilon^{2}\left\|u_{0}\right\|^{2}+\mu\left\|\nabla^{2 m} u_{0}\right\|^{2}+\frac{2(\varepsilon+1)}{\rho+2}\left\|u_{0}\right\|_{L^{\rho+2}(\Omega)}^{\rho+2}\right) \mathrm{e}^{-\alpha_{1} t}+\frac{C_{0}}{\alpha_{1}}\left(1-\mathrm{e}^{-\alpha_{1} t}\right), \\
\frac{\beta}{2} \int_{0}^{T}\left\|\nabla^{2 m} v\right\|^{2} \mathrm{~d} t \leq C_{0} T+y_{1}(0) .
\end{gathered}
$$

where $\alpha_{1}=\min \left\{2 \varepsilon, \frac{a_{1}}{\mu}, a_{2}, \frac{\varepsilon(\rho+2)}{\varepsilon+1}\right\}$,

$y_{1}(0)=\left\|v_{0}\right\|^{2}+\varepsilon^{2}\left\|u_{0}\right\|^{2}+\mu\left\|\nabla^{2 m} u_{0}\right\|^{2}+\frac{2(\varepsilon+1)}{\rho+2}\left\|u_{0}\right\|_{L^{\rho+2}(\Omega)}^{\rho+2}$.

So there is a non-negative real number $C\left(R_{1}\right)$ and $t=t_{1}(\Omega)>0$, make

$$
\|(u, v)\|_{E_{0}}^{2} \leq \frac{2 C_{0}}{\alpha_{1}}=C\left(R_{1}\right),\left(t>t_{1}\right) .
$$

Proof. Set $v=u_{t}+\varepsilon u$, take the inner product of both sides of Equation (1.1) 
with $v$ in $H$, we obtain

$$
\begin{gathered}
\left(u_{t t}+M\left(\left\|\nabla^{m} u\right\|_{p}^{p}\right)(-\Delta)^{2 m} u+\beta(-\Delta)^{2 m} u_{t}+|u|^{\rho}\left(u_{t}+u\right), v\right)=(f(x), v) . \\
\left(u_{t t}, v\right)=\frac{1}{2} \frac{\mathrm{d}}{\mathrm{d} t}\|v\|^{2}+\varepsilon^{2} \frac{1}{2} \frac{\mathrm{d}}{\mathrm{d} t}\|u\|^{2}-\varepsilon\|v\|^{2}+\varepsilon^{3}\|u\|^{2} .
\end{gathered}
$$

According to hypothesis $\left(\mathrm{H}_{1}\right)$, we can get

$$
\begin{aligned}
\left(M\left(\left\|\nabla^{m} u\right\|_{p}^{p}\right)(-\Delta)^{2 m} u, v\right) & =\left(M\left(\left\|\nabla^{m} u\right\|_{p}^{p}\right)(-\Delta)^{2 m} u, u_{t}+\varepsilon u\right) \\
& \geq \frac{\mu}{2} \frac{\mathrm{d}}{\mathrm{d} t}\left\|\nabla^{2 m} u\right\|^{2}+\varepsilon \mu_{0}\left\|\nabla^{2 m} u\right\|^{2} .
\end{aligned}
$$

By Young's inequality and Poincarês inequality

$$
\begin{aligned}
\left(\beta(-\Delta)^{2 m} u_{t}, v\right) & =\beta\left\|\nabla^{2 m} v\right\|^{2}-\varepsilon \beta\left(\nabla^{2 m} u, \nabla^{2 m} v\right) \\
& \geq \beta\left\|\nabla^{2 m} v\right\|^{2}-\beta\left(\varepsilon\left\|\nabla^{2 m} u\right\|\left\|\nabla^{2 m} v\right\|\right) \\
& \geq \frac{\beta}{2}\left\|\nabla^{2 m} v\right\|^{2}-\frac{\varepsilon^{2}}{2}\left\|\nabla^{2 m} u\right\|^{2} .
\end{aligned}
$$

where $\lambda_{1}$ is the first eigenvalue of $-\Delta$ with homogeneous Dirichlet boundary condition on $\Omega$.

we can get

$$
\begin{aligned}
\left(|u|^{\rho}\left(u_{t}+u\right), v\right) & =\left(|u|^{\rho}, u_{t}^{2}\right)+\varepsilon\left(|u|^{\rho} u_{t}, u\right)+\left(|u|^{\rho} u, u_{t}\right)+\varepsilon\left(|u|^{\rho} u, u\right) \\
& \geq \varepsilon \int_{\Omega}|u|^{\rho+2} \mathrm{~d} x+(\varepsilon+1) \int_{\Omega}|u|^{\rho} u \cdot u_{t} \mathrm{~d} x \\
& \geq \varepsilon\|u\|_{L^{\rho+2}(\Omega)}^{\rho+2}+\frac{\varepsilon+1}{\rho+2} \frac{\mathrm{d}}{\mathrm{d} t}\|u\|_{L^{\rho+2}(\Omega)}^{\rho+2} .
\end{aligned}
$$

According to Young's inequality, we get

$$
(f(x), v) \leq\|f\|\|v\| \leq \frac{\varepsilon^{2}}{2}\|v\|^{2}+\frac{1}{2 \varepsilon^{2}}\|f\|^{2} .
$$

Substitute (2.5)-(2.9) into (2.4),

$$
\begin{aligned}
& \frac{\mathrm{d}}{\mathrm{d} t}\left(\mu\left\|\nabla^{2 m} u\right\|^{2}+\|v\|^{2}+\varepsilon^{2}\|u\|^{2}+\frac{2(\varepsilon+1)}{\rho+2}\|u\|_{L^{\rho+2}(\Omega)}^{\rho+2}\right)+\left(2 \varepsilon \mu_{0}-\varepsilon^{2}\right)\left\|\nabla^{2 m} u\right\|^{2} \\
& +\frac{\beta}{2}\left\|\nabla^{2 m} v\right\|^{2}+\left(\frac{\beta \lambda_{1}^{2 m}}{2}-2 \varepsilon-\varepsilon^{2}\right)\|v\|^{2}+2 \varepsilon^{3}\|u\|^{2}+2 \varepsilon\|u\|_{L^{\rho+2}(\Omega)}^{\rho+2} \leq \frac{1}{\varepsilon^{2}}\|f\|^{2}=C_{0} .
\end{aligned}
$$

Make $a_{1}=2 \varepsilon \mu_{0}-\varepsilon^{2} \geq 0, a_{2}=\frac{\beta \lambda_{1}^{2 m}}{2}-2 \varepsilon-\varepsilon^{2} \geq 0$.

Take $\alpha_{1}=\min \left\{2 \varepsilon, \frac{a_{1}}{\mu}, a_{2}, \frac{(\rho+2) \varepsilon}{\varepsilon+1},\right\}$.

Then (2.10) can be converted into

$$
\begin{aligned}
& \frac{\mathrm{d}}{\mathrm{d} t}\left(\mu\left\|\nabla^{2 m} u\right\|^{2}+\|v\|^{2}+\varepsilon^{2}\|u\|^{2}+\frac{2(\varepsilon+1)}{\rho+2}\|u\|_{L^{\rho+2}(\Omega)}^{\rho+2}\right) \\
& +\alpha_{1}\left(\mu\left\|\nabla^{2 m} u\right\|^{2}+\|v\|^{2}+\varepsilon^{2}\|u\|^{2}+\frac{2(\varepsilon+1)}{\rho+2}\|u\|_{L^{\rho+2}(\Omega)}^{\rho+2}\right)+\frac{\beta}{2}\left\|\nabla^{2 m} v\right\|^{2} \leq C_{0} .
\end{aligned}
$$


By Gronwalls inequality

$$
\begin{gathered}
\left\|\nabla^{2 m} u\right\|^{2}+\|v\|^{2} \\
\leq\left(\left\|v_{0}\right\|^{2}+\varepsilon^{2}\left\|u_{0}\right\|^{2}+\mu\left\|\nabla^{2 m} u_{0}\right\|^{2}+\frac{2(\varepsilon+1)}{\rho+2}\left\|u_{0}\right\|_{L^{\rho+2}(\Omega)}^{\rho+2}\right) \mathrm{e}^{-\alpha_{1} t}+\frac{C_{0}}{\alpha_{1}}\left(1-\mathrm{e}^{-\alpha_{1} t}\right), \\
\frac{\beta}{2} \int_{0}^{T}\left\|\nabla^{2 m} v\right\|^{2} \mathrm{~d} t \leq C_{0} T+y_{1}(0) .
\end{gathered}
$$

where $y_{1}=\|v\|^{2}+\varepsilon^{2}\|u\|^{2}+\mu\left\|\nabla^{2 m} u\right\|^{2}+\frac{2(\varepsilon+1)}{\rho+2}\|u\|_{L^{\rho+2}(\Omega)}^{\rho+2}$.

So there is a non-negative real number $C\left(R_{1}\right)$ and $t=t_{1}(\Omega)>0$, such as

$$
\|(u, v)\|_{E_{0}}^{2} \leq \frac{2 C_{0}}{\alpha_{1}}=C\left(R_{1}\right),\left(t>t_{1}\right) \text {. }
$$

Lemma 2.1 is proved.

Lemma 2.2 Assume that (H1), (H2) holds, if $f(x) \in H$, $\left(u_{0}, v_{0}\right) \in E_{k}(k=1,2, \cdots, 2 m), v=u_{t}+\varepsilon u, \varepsilon>0$, Then the smooth solution $(u, v) \in E_{k},(k=1,2, \cdots, 2 m)$ of the initial-boundary value problem (1.1)-(1.3) satisfies $u \in L^{\infty}\left(0,+\infty ; H_{0}^{2 m+k}(\Omega)\right)$,

$v \in L^{\infty}\left(0,+\infty ; H^{k}(\Omega)\right) \cap L^{2}\left(0, T ; H_{0}^{2 m+k}(\Omega)\right)$ and satisfy that following inequality

$$
\begin{aligned}
&\|(u, v)\|_{E_{k}}^{2}=\left\|\nabla^{2 m+k} u\right\|^{2}+\left\|\nabla^{k} v\right\|^{2} \\
& \leq\left(\left\|\nabla^{k} v_{0}\right\|^{2}+(\mu-\beta \varepsilon)\left\|\nabla^{2 m+k} u_{0}\right\|^{2}\right) \mathrm{e}^{-\alpha_{2} t}+\frac{C_{3}}{\alpha_{2}}\left(1-\mathrm{e}^{-\alpha_{2} t}\right), \\
& \frac{\beta}{6} \int_{0}^{T}\left\|\nabla^{2 m+k} v\right\|^{2} \mathrm{~d} t \leq y_{2}(0)+C_{3} T .
\end{aligned}
$$

So there is a non-negative real number $C\left(R_{2}\right)$ and $t=t_{2}(\Omega)>0$, make

$$
\|(u, v)\|_{E_{k}}^{2} \leq \frac{2 C_{3}}{\alpha_{2}}=C\left(R_{2}\right),\left(t>t_{2}\right) .
$$

Proof. Take the inner product of $(-\Delta)^{k} v=(-\Delta)^{k} u_{t}+(-\Delta)^{k} \varepsilon u$ and the two sides of Equation (1.1), and get

$$
\begin{aligned}
& \left(u_{t t}+M\left(\left\|\nabla^{m} u\right\|_{p}^{p}\right)(-\Delta)^{2 m} u+\beta(-\Delta)^{2 m} u_{t}+|u|^{\rho}\left(u_{t}+u\right),(-\Delta)^{k} v\right) \\
& =\left(f(x),(-\Delta)^{k} v\right) .
\end{aligned}
$$

By Young's inequality, Poincarês inequality

$$
\left(u_{t t},(-\Delta)^{k} v\right) \geq \frac{1}{2} \frac{\mathrm{d}}{\mathrm{d} t}\left\|\nabla^{k} v\right\|^{2}-\frac{2 \varepsilon+\varepsilon^{2}}{2}\left\|\nabla^{k} v\right\|^{2}-\frac{\varepsilon^{2}}{2 \lambda_{1}^{2 m}}\left\|\nabla^{2 m+k} u\right\|^{2} .
$$

According to hypothesis (H1)

$$
\left(M\left(\left\|\nabla^{m} u\right\|_{p}^{p}\right),(-\Delta)^{k} v\right) \geq \frac{\mu}{2} \frac{\mathrm{d}}{\mathrm{d} t}\left\|\nabla^{2 m+k} u\right\|^{2}+\varepsilon \mu_{0}\left\|\nabla^{2 m+k} u\right\|^{2} .
$$

By Young's inequality, Poincarês inequality 


$$
\begin{aligned}
\left(\beta(-\Delta)^{2 m} u_{t},(-\Delta)^{k} v\right) & =\beta\left\|\nabla^{2 m+k} v\right\|^{2}-\beta \varepsilon\left\|\nabla^{2 m+k} u\right\|\left\|\nabla^{2 m+k} v\right\| \\
& \geq \frac{\beta}{6}\left\|\nabla^{2 m+k} v\right\|^{2}-\frac{\beta \varepsilon^{2}}{2}\left\|\nabla^{2 m+k} u\right\|^{2}+\frac{\beta \lambda_{1}^{2 m}}{3}\left\|\nabla^{k} v\right\|^{2} .
\end{aligned}
$$

where $\lambda_{1}$ is the first eigenvalue of $-\Delta$ with homogeneous Dirichlet boundary condition on $\Omega$.

we can get

$$
\begin{aligned}
& \left(|u|^{\rho}\left(u_{t}+u\right),(-\Delta)^{k} v\right) \\
& =\left(|u|^{\rho} v,(-\Delta)^{k} v\right)-\varepsilon\left(|u|^{\rho} u,(-\Delta)^{k} v\right)+\left(|u|^{\rho} u,(-\Delta)^{k} v\right) \\
& \geq(1-\varepsilon)\left(|u|^{\rho} u,(-\Delta)^{k} v\right),
\end{aligned}
$$

By Young's inequality, Poincarês inequality

$$
\begin{aligned}
(1-\varepsilon)\left(|u|^{\rho} u,(-\Delta)^{k} v\right) & \leq(1-\varepsilon)\left\|\nabla^{2 k} v\right\|\left(\int_{\Omega}|u|^{2 \rho+2} \mathrm{~d} x\right)^{\frac{1}{2}}, \\
& \leq C_{1}\left\|\nabla^{2 k} v\right\| \leq \frac{\beta}{12}\left\|\nabla^{2 m+k} v\right\|^{2}+C_{2} .
\end{aligned}
$$

By Schwarz's inequality, Young's inequality

$$
\left(f(x),(-\Delta)^{k} v\right) \leq \frac{\varepsilon^{2}}{2}\left\|\nabla^{k} v\right\|^{2}+\frac{1}{2 \varepsilon^{2}}\left\|\nabla^{k} f(x)\right\|^{2} .
$$

Substitute (2.19)-(2.23) into (2.18) to get

$$
\begin{aligned}
& \frac{\mathrm{d}}{\mathrm{d} t}\left(\left\|\nabla^{k} v\right\|^{2}+\mu\left\|\nabla^{2 m+k} u\right\|^{2}\right)+\left(\frac{2 \beta \lambda_{1}^{2 m}}{3}-2 \varepsilon-2 \varepsilon^{2}\right)\left\|\nabla^{k} v\right\|^{2} \\
& +\left(2 \varepsilon \mu_{0}-\frac{\varepsilon^{2}}{\lambda_{1}^{2 m}}-\beta \varepsilon^{2}\right)\left\|\nabla^{2 m+k} u\right\|^{2}+\frac{\beta}{6}\left\|\nabla^{2 m+k} v\right\|^{2} \leq C_{3} .
\end{aligned}
$$

Take $a_{3}=\frac{2 \beta \lambda_{1}^{2 m}}{3}-2 \varepsilon-2 \varepsilon^{2} \geq 0, a_{4}=2 \varepsilon \mu_{0}-\frac{\varepsilon^{2}}{\lambda_{1}^{2 m}}-\beta \varepsilon^{2} \geq 0$.

Let $\alpha_{2}=\min \left\{\frac{2 \beta \lambda_{1}^{2 m}}{3}-2 \varepsilon-2 \varepsilon^{2}, \frac{2 \varepsilon \mu_{0}-\frac{\varepsilon^{2}}{\lambda_{1}^{2 m}}-\beta \varepsilon^{2}}{\mu}\right\}$.

Then (2.24) can be converted into

$$
\frac{\mathrm{d}}{\mathrm{d} t}\left(\left\|\nabla^{k} v\right\|^{2}+\mu\left\|\nabla^{2 m+k} u\right\|^{2}\right)+\alpha_{2}\left(\left\|\nabla^{k} v\right\|^{2}+\mu\left\|\nabla^{2 m+k} u\right\|^{2}\right)+\frac{\beta}{6}\left\|\nabla^{2 m+k} v\right\|^{2} \leq C_{3} .
$$

By Gronwalls inequality

$$
\begin{gathered}
\left\|\nabla^{2 m+k} u\right\|^{2}+\left\|\nabla^{k} v\right\|^{2} \leq\left(\mu\left\|\nabla^{2 m+k} u_{0}\right\|^{2}+\left\|\nabla^{k} v_{0}\right\|^{2}\right) \mathrm{e}^{-\alpha_{2} t}+\frac{C_{3}}{\alpha_{2}}\left(1-\mathrm{e}^{-\alpha_{2} t}\right), \\
\frac{\beta_{6}^{T}}{\int_{0}^{T}}\left\|\nabla^{2 m+k} v\right\|^{2} \mathrm{~d} t \leq y_{2}(0)+C_{3} T .
\end{gathered}
$$

where $y_{2}=\left\|\nabla^{k} v\right\|^{2}+\mu\left\|\nabla^{2 m+k} u\right\|^{2}$. 
So there is a non-negative real number $C\left(R_{2}\right)$ and $t=t_{2}(\Omega)>0$, make

$$
\|(u, v)\|_{E_{k}}^{2} \leq \frac{2 C_{3}}{\alpha_{2}}=C\left(R_{2}\right),\left(t>t_{2}\right)
$$

Lemma 2.2 is proved.

Theorem 2.1 Under the assumption of lemma 2.1 and lemma 2.2, and satisfy the hypothesis (H1), (H2), Then the initial-boundary value problem (1.1)-(1.3) has a unique smooth solution $(u, v) \in L^{\infty}\left(0,+\infty ; E_{k}\right), v \in L^{2}\left(0, T ; H_{0}^{2 m+k}(\Omega)\right)$, $(k=0,1,2, \cdots, 2 m)$.

Proof. Existence: Galerkin's method is used to prove the existence of global smooth solution.

Step 1: construct an approximate solution.

Let $(-\Delta)^{2 m+k} w_{j}=\lambda_{j} w_{j}, k=0,1,2, \cdots, 2 m$. where $\lambda_{j}$ is the eigenvalue of $-\Delta$ with homogeneous Dirichlet boundary on $\Omega, w_{j}$ denotes the eigenfunction determined by the corresponding eigenvalue $\lambda_{j}, w_{1}, \cdots, w_{n}$ constitute the orthonormal basis of $H$ from the eigenvalue theory.

Let the approximate solution of the problem (1.1)-(1.3) be $u_{s}=u_{s}(t)=\sum_{j=1}^{s} g_{j s}(t) w_{j}$, where $g_{j s}(t)$ is determined by the following equations.

$$
\begin{gathered}
\left(u_{s t t}+M\left(\left\|\nabla^{m} u_{s}\right\|_{p}^{p}\right)(-\Delta)^{2 m} u_{s}+\beta(-\Delta)^{2 m} u_{s t}+\left|u_{s}\right|^{\rho}\left(u_{s t}+u_{s}\right), w_{j}\right)=\left(f(x), w_{j}\right) . \\
(1 \leq j \leq s)
\end{gathered}
$$

The formula (2.29) satisfies the initial condition $u_{s}(0)=u_{0 s}, u_{s t}(0)=u_{1 s}$.

When $s \rightarrow+\infty,\left(u_{0 s}, u_{1 s}\right) \rightarrow\left(u_{0}, u_{1}\right)$ in $E_{k}$, according to the basic theory of ordinary differential equations, the approximate solution $u_{s}(t)$ exists on $\left(0, t_{s}\right)$.

Step 2: Prior estimation.

$v_{s}(t)=u_{s t}(t)+\varepsilon u_{s}(t)$, multiplying by $g_{j s}^{\prime}(t)+\varepsilon g_{j s}(t)$ and summing over $j$, we can get

1) $k=0$, by lemma 2.1 , there is

$$
\begin{gathered}
\left\|\left(u_{s}, v_{s}\right)\right\|_{E_{0}}^{2} \leq y_{1}(0) \mathrm{e}^{-\alpha_{1} t}+\frac{C_{0}}{\alpha_{1}}\left(1-\mathrm{e}^{-\alpha_{1} t}\right), \\
\frac{\beta}{2} \int_{0}^{T}\left\|\nabla^{2 m} v\right\|^{2} \mathrm{~d} t \leq C_{0} T+y_{1}(0) .
\end{gathered}
$$

2) $k=1,2, \cdots, 2 m$, by lemma 2.2 , there is

$$
\begin{gathered}
\left\|\left(u_{s}, v_{s}\right)\right\|_{E_{k}}^{2} \leq y_{2}(0) \mathrm{e}^{-\alpha_{2} t}+\frac{C_{3}}{\alpha_{2}}\left(1-\mathrm{e}^{-\alpha_{2} t}\right), \\
\frac{\beta}{6} \int_{0}^{T}\left\|\nabla^{2 m+k} v\right\|^{2} \mathrm{~d} t \leq y_{2}(0)+C_{3} T .
\end{gathered}
$$

From (2.30) and (2.32), $\left(u_{s}, v_{s}\right)$ is bounded in $L^{\infty}\left(0,+\infty ; E_{k}\right),\left(u_{s}, v_{s}\right)$ in $L^{\infty}\left(0,+\infty ; E_{0}\right)$ is bounded. 
It can be seen that the formula (2.30)-(2.33) holds a priori estimates for lemma 2.1 and lemma 2.2 respectively.

Step 3: Limit process.

In $E_{k}(k=0,1,2, \cdots, 2 m)$ space, select the subsequence $\left\{u_{h}\right\}$ from the sequence $\left\{u_{s}\right\}$,

Make $\left(u_{h}, v_{h}\right) \rightarrow(u, v)$ in $L^{\infty}\left(0,+\infty ; E_{k}\right)$ weak * convergence.

According to Rellich-Kondrachov compact embedding theorem, $E_{k}$ compactly embeds $E_{0}$, Then $\left(u_{h}, v_{h}\right) \rightarrow(u, v)$ converges strongly almost everywhere in $E_{0}$.

Let $s=h$ in (2.29), and take the limit, for fixed $j, h \geq j$,

Then from (2.29), make $u_{s} \rightarrow u$ in $L^{\infty}\left(0,+\infty ; H_{0}^{2 m+k}(\Omega)\right)$ weak * convergence.

Thus $\left(u_{h}(t),(-\Delta)^{k} w_{j}\right) \rightarrow\left(u(t), \lambda_{j}^{k} w_{j}\right)$ in $L^{\infty}(0,+\infty)$ weak ${ }^{\star}$ convergence. $\left(u_{h t}(t),(-\Delta)^{k} w_{j}\right) \rightarrow\left(u_{t}(t), \lambda_{j}^{k} w_{j}\right)$ in $L^{\infty}(0,+\infty)$ weak ${ }^{\star}$ convergence.

So $\left(u_{h t t},(-\Delta)^{k} w_{j}\right)=\frac{\mathrm{d}}{\mathrm{d} t}\left(u_{h t},(-\Delta)^{k} w_{j}\right) \rightarrow\left(u_{h t}, \lambda_{j}^{k} w_{j}\right)$ in $D^{\prime}[0,+\infty)$ convergence.

$D^{\prime}[0,+\infty)$ is a conjugate space of $D[0,+\infty)$ infinite differentiable space.

$\left(M\left(\left\|\nabla^{m} u_{h}\right\|_{p}^{p}\right)(-\Delta)^{k} u_{h},(-\Delta)^{k} w_{j}\right) \rightarrow\left(M\left(\left\|\nabla^{m} u_{h}\right\|_{p}^{p}\right)(-\Delta)^{\frac{2 m+k}{2}} u, \lambda_{j}^{\frac{2 m+k}{2}} w_{j}\right) \quad$ in

$L^{\infty}(0,+\infty)$ weak * convergence.

$$
\left(\beta(-\Delta)^{2 m} u_{h t},(-\Delta)^{k} w_{j}\right) \rightarrow \beta\left((-\Delta)^{\frac{k}{2}} v, \lambda_{j}^{\frac{2 m+k}{2}} w_{j}\right)-\varepsilon \beta\left((-\Delta)^{\frac{2 m+k}{2}} u, \lambda_{j}^{\frac{2 m+k}{2}} w_{j}\right)
$$

in $L^{\infty}(0,+\infty)$ weak * convergence.

$u_{h t} \rightarrow u_{t}$ in $E_{0}$ strong convergence, almost everywhere convergence.

$$
\left|u_{h}\right|^{\rho}\left(u_{h t}+u_{h}\right) \rightarrow w .
$$

In $L^{\infty}\left(0,+\infty ; L^{\frac{\rho+2}{\rho+1}}(\Omega)\right)$ weak * convergence.

Take $D=\Omega, g_{h}=\left|u_{h}\right|^{\rho}\left(u_{h t}+u_{h}\right)$. (2.34) almost everywhere $g_{h} \rightarrow|u|^{\rho}\left(u_{t}+u\right), \quad g_{h} \rightarrow w$ in $L^{\frac{\rho+2}{\rho+1}}(\Omega)$ weak convergence.

Therefore $w=g=|u|^{\rho}\left(u_{t}+u\right)$, so $\left(\left|u_{h}\right|^{\rho}\left(u_{h t}+u_{h}\right),(-\Delta)^{k} w_{j}\right) \rightarrow\left(|u|^{\rho}\left(u_{t}+u\right),(-\Delta)^{k} w_{j}\right) \quad$ in $\quad L^{\infty}(0,+\infty)$ weak * convergence. In particular, $u_{0 h} \rightarrow u_{0}$ weak convergence in the $E_{k}, u_{1 h} \rightarrow u_{1}$ weak convergence in $E_{k}$, From all $j$ and $h \rightarrow+\infty$,it can be introduced

$$
\begin{aligned}
& \left(u_{t t}+M\left(\left\|\nabla^{m} u\right\|_{p}^{p}\right)(-\Delta)^{2 m} u+\beta(-\Delta)^{2 m} u_{t}+|u|^{\rho}\left(u_{t}+u\right),(-\Delta)^{k} w_{j}\right) \\
& =\left(f(x),(-\Delta)^{k} w_{j}\right) .
\end{aligned}
$$

Because of the density of $w_{1}, w_{2}, \cdots, w_{k}, \cdots$. 


$$
\begin{aligned}
& \left(u_{t t}+M\left(\left\|\nabla^{m} u\right\|_{p}^{p}\right)(-\Delta)^{2 m} u+\beta(-\Delta)^{2 m} u_{t}+|u|^{\rho}\left(u_{t}+u\right), v\right) \\
& =(f(x), v), \forall v \in H_{0}^{2 m+k}(\Omega) .
\end{aligned}
$$

Therefore, the existence is proved.

The uniqueness of the solution.

Set $u^{*}, v^{*}$ is equations of two solutions, make $w=u^{*}-v^{*}, w$ satisfies

$$
\begin{gathered}
w_{t t}+M\left(\left\|\nabla^{m} u^{*}\right\|_{p}^{p}\right)(-\Delta)^{2 m} u^{*}-M\left(\left\|\nabla^{m} v^{*}\right\|_{p}^{p}\right)(-\Delta)^{2 m} v^{*}+\beta(-\Delta)^{2 m} w_{t} \\
=\left|v^{*}\right|^{\rho}\left(v_{t}^{*}+v^{*}\right)-\left|u^{*}\right|^{\rho}\left(u_{t}^{*}+u^{*}\right), \\
w(0)=0, w^{\prime}(0)=0, x \in \Omega \subset R^{n} .
\end{gathered}
$$

Take inner product of (2.35) and $w_{t}$ in $H$ is as follows

$$
\begin{aligned}
& \left(w_{t t}+M\left(\left\|\nabla^{m} u^{*}\right\|_{p}^{p}\right)(-\Delta)^{2 m} u^{*}-M\left(\left\|\nabla^{m} v^{*}\right\|_{p}^{p}\right)(-\Delta)^{2 m} v^{*}+\beta(-\Delta)^{2 m} w_{t}, w_{t}\right) \\
& =\left(\left|v^{*}\right|^{\rho}\left(v_{t}^{*}+v^{*}\right)-\left|u^{*}\right|^{\rho}\left(u_{t}^{*}+u^{*}\right), w_{t}\right) .
\end{aligned}
$$

Therefore

$$
\begin{gathered}
\left(w_{t t}, w_{t}\right)=\frac{1}{2} \frac{\mathrm{d}}{\mathrm{d} t}\left\|w_{t}\right\|^{2}, \\
\left(\beta(-\Delta)^{2 m} w_{t}, w_{t}\right)=\beta\left\|\nabla^{2 m} w_{t}\right\|^{2} .
\end{gathered}
$$

By differential mean value theorem, Young's inequality

$$
\begin{aligned}
& \left(M\left(\left\|\nabla^{m} u^{*}\right\|_{p}^{p}\right)(-\Delta)^{2 m} u^{*}-M\left(\left\|\nabla^{m} v^{*}\right\|_{p}^{p}\right)(-\Delta)^{2 m} v^{*}, w_{t}\right) \\
& \geq \frac{1}{2} M\left(\left\|\nabla^{m} u^{*}\right\|_{p}^{p}\right) \frac{\mathrm{d}}{\mathrm{d} t}\left\|\nabla^{2 m} w\right\|^{2}-\left(M^{\prime}\left(\xi_{1}\right)\left(\left\|\nabla^{m} u^{*}\right\|_{p}^{p}-\left\|\nabla^{m} v^{*}\right\|_{p}^{p}\right)(-\Delta)^{2 m} v^{*}, w_{t}\right) \\
& \geq \frac{\mu}{2} \frac{\mathrm{d}}{\mathrm{d} t}\left\|\nabla^{2 m} w\right\|^{2}-\frac{\beta}{2}\left\|\nabla^{2 m} w_{t}\right\|^{2}-\frac{C_{4}^{2}}{2 \beta}\left\|\nabla^{2 m} w\right\|^{2} .
\end{aligned}
$$

where $\xi_{1}=\delta u^{*}+(1-\delta) v^{*}, 0<\delta<1$.

By Young's inequality

$$
\begin{aligned}
& \left(\left|v^{*}\right|^{\rho}\left(v_{t}^{*}+v^{*}\right)-\left|u^{*}\right|^{\rho}\left(u_{t}^{*}+u^{*}\right), w_{t}\right) \\
& \leq C_{5}\left\|w_{t}\right\|^{2} \int_{\Omega}\left(\left|v^{*}\right|^{\rho}+\left|u^{*}\right|^{\rho}\right) \mathrm{d} x+C_{6}\|w\|\left\|w_{t} \mid\right\| \int_{\Omega}\left(\left|v^{*}\right|^{\rho}+\left|u^{*}\right|^{\rho}\right) \mathrm{d} x \\
& \leq C_{5}\left(\left\|\left.v^{*}\right|^{\rho}\right\|_{L^{\infty}(\Omega)}+\left\|\left.u^{*}\right|^{\rho}\right\|_{L^{\infty}(\Omega)}\right)\left\|w_{t}\right\|^{2} \\
& +\frac{C_{6}\left(\left.\left\|v^{*}\right\|^{\rho}\left\|_{L^{\infty}(\Omega)}+\right\| u^{*}\right|^{\rho} \|_{L^{\infty}(\Omega)}\right)}{2}\left\|w_{t}\right\|^{2}+\frac{C_{6}\left(\left\|\left.v^{*}\right|^{\rho}\right\|_{L^{\infty}(\Omega)}+\left\|\left.u^{*}\right|^{\rho}\right\|_{L^{\infty}(\Omega)}\right)}{2}\|w\|^{2}
\end{aligned}
$$

by the interpolation inequality

$$
\left\|v^{*}\right\|_{\infty}^{\rho} \leq C_{7}\left\|\nabla^{2 m} v^{*}\right\|^{\frac{\rho n}{4 m}}
$$

in the same way with 


$$
\left\|u^{*}\right\|_{\infty}^{\rho} \leq C_{8}\left\|\nabla^{2 m} u^{*}\right\|^{\frac{\rho n}{4 m}},
$$

where $\rho \leq \frac{8 m}{n}$.

By Poincarês inequality

$$
\begin{aligned}
& \left(\left|v^{*}\right|^{\rho}\left(v_{t}^{*}+v^{*}\right)-\left|u^{*}\right|^{\rho}\left(u_{t}^{*}+u^{*}\right), w_{t}\right) \\
& \leq C_{9}\left(\left\|\nabla^{2 m} v_{t}^{*}\right\|^{\frac{\rho n}{4 m}}+\left\|\nabla^{2 m} u_{t}^{*}\right\|^{\frac{\rho n}{4 m}}\right)\left\|w_{t}\right\|^{2}+C_{10}\left(\left\|\nabla^{2 m} v_{t}^{*}\right\| \frac{\rho n}{4 m}+\left\|\nabla^{2 m} u_{t}^{*}\right\| \frac{\rho n}{4 m}\right)\|w\|^{2} \\
& \leq C_{9}\left(\left\|\nabla^{2 m} v_{t}^{*}\right\| \frac{\rho n}{4 m}+\left\|\nabla^{2 m} u_{t}^{*}\right\| \frac{\rho n}{4 m}\right)\left\|w_{t}\right\|^{2}+\frac{C_{10}}{\lambda_{1}^{2 m}}\left(\left\|\nabla^{2 m} v_{t}^{*}\right\| \frac{\rho n}{4 m}+\left\|\nabla^{2 m} u_{t}^{*}\right\| \frac{\rho n}{4 m}\right)\left\|\nabla^{2 m} w\right\|^{2} .
\end{aligned}
$$

By (2.37)-(2.40)

$$
\begin{aligned}
\frac{\mathrm{d}}{\mathrm{d} t}\left(\left\|w_{t}\right\|^{2}+\mu\left\|\nabla^{2 m} w\right\|^{2}\right) \leq & 2 C_{9}\left(\left\|\nabla^{2 m} v_{t}^{*}\right\| \frac{\rho n}{4 m}+\left\|\nabla^{2 m} u_{t}^{*}\right\| \frac{\rho n}{4 m}\right)\left\|w_{t}\right\|^{2} \\
& +\left(\frac{C_{4}^{2}}{\beta}+\frac{2 C_{10}\left(\left\|\nabla^{2 m} v_{t}^{*}\right\| \frac{\rho n}{4 m}+\left\|\nabla^{2 m} u_{t}^{*}\right\| \frac{\rho n}{4 m}\right)}{\lambda_{1}^{2 m}}\right)\left\|\nabla^{2 m} w\right\|^{2}
\end{aligned}
$$

Take the

$$
\alpha_{3}=2 C_{9}\left(\left\|\nabla^{2 m} v_{t}^{*}\right\|^{\frac{\rho n}{4 m}}+\left\|\nabla^{2 m} u_{t}^{*}\right\|^{\frac{\rho n}{4 m}}\right)+\frac{\frac{C_{4}^{2}}{\beta}+\frac{2 C_{10}\left(\left\|\nabla^{2 m} v_{t}^{*}\right\|^{\frac{\rho n}{4 m}}+\left\|\nabla^{2 m} u_{t}^{*}\right\|^{\frac{\rho n}{4 m}}\right)}{\lambda_{1}^{2 m}}}{\mu},
$$

there are

$$
\frac{\mathrm{d}}{\mathrm{d} t} y_{3}(t) \leq \alpha_{3}(t) y_{3}(t) .
$$

where $y_{3}(t)=\left\|w_{t}\right\|^{2}+\mu\left\|\nabla^{2 m} w\right\|^{2}$.

By Gronwalls inequality

$$
y_{3}(t) \leq y_{3}(0) \mathrm{e}^{\iint^{T} \alpha_{3}(t) \mathrm{d} t}=0 .
$$

Thus $y_{3}(t)=0$, or $u^{*}=v^{*}$, therefore, the uniqueness is proved.

Theorem 2.2 [11] Let $E$ be a Banach space and $S(t): E \rightarrow E$ semigroups satisfy the following conditions.

1) semigroup $S(t)$ is uniformly bounded in $E$, and $\forall R>0$, there is a constant $C(R)$, so that when $\|u\|_{E} \leq R$, there is

$$
\|S(t) u\|_{E} \leq C(R) \cdot(\forall t \in[0, \infty)) .
$$

2) There is a bounded absorption set $B_{0}$ in $E$.

3) $S(t)(t>0)$ is a fully continuous operator

Then a semigroup $S(t)$ is said to have a compact global attractor $A_{0}$.

Theorem 2.2 in Banach space $E$ change to the Hilbert space $E_{k}$, has the 
following the existence theorem of the family of global attractor.

Theorem 2.3 If the global smooth solution of the problem (1.1)-(1.3) satisfies the assumptions and conditions of lemma 2.1 and lemma 2.2, then the problem (1.1)-(1.3) have a family of global attractor $A_{k},(k=1,2, \cdots, 2 m)$. That is, there is a compact set $A_{k}$, which makes.

1) $S(t) A_{k}=A_{k},(t>0)$.

2) $\lim _{t \rightarrow \infty} \operatorname{dist}\left(S(t) B_{0 k}, A_{k}\right)=0,\left(\forall B_{0 k} \subset E_{k}\right)$.

where $\operatorname{dist}\left(S(t) B_{0 k}, A_{k}\right)=\sup _{x \in B_{0 k}} \inf _{y \in A_{k}}\|S(t) x-y\|_{E_{k}}, \quad S(t)$ is the solution semigroup of (1.1)-(1.3).

Proof. It is necessary to verify the hypothesis (1), (2), (3) of theorem 2.2. It is easy to know that the Equation (1.1) has a solution semigroup $S(t): E_{k} \rightarrow E_{k}$ under the hypothesis of theorem 2.3.

1) by lemma 2.1, lemma 2.2, bounded set for $\forall B_{0 k} \subset E_{k}$ and contained in

$$
\begin{aligned}
& \left\{\|(u, v)\|_{E_{k}} \leq R_{k}\right\} . \\
& \quad\left\|S(t)\left(u_{0}, v_{0}\right)\right\|_{E_{k}}^{2}=\|u\|_{H_{0}^{2 m+k}(\Omega)}^{2}+\|v\|_{H_{0}^{k}(\Omega)}^{2} \leq\left\|u_{0}\right\|_{H_{0}^{2 m+k}(\Omega)}^{2}+\left\|v_{0}\right\|_{H_{0}^{k}(\Omega)}^{2} \leq R_{k}^{2} .
\end{aligned}
$$

where $t \geq 0,\left(u_{0}, v_{0}\right) \in B_{0 k}$, this suggests that the $\{S(t)\}(t \geq 0)$ in $E_{k}$ uniformly bounded.

2) by lemma 2.1, lemma 2.2, there are further

$$
\left\|S(t)\left(u_{0}, v_{0}\right)\right\|_{E_{k}}^{2}=\|u\|_{H_{0}^{2 m+k}(\Omega)}^{2}+\|v\|_{H_{0}^{k}(\Omega)}^{2} \leq R_{k}^{2}, t \geq t_{0}=t_{0}\left(R_{k}\right) .
$$

So $B_{0 k}=\left\{(u, v) \in E_{k}:\|u\|_{H_{0}^{2 m+k}(\Omega)}^{2}+\|v\|_{H_{0}^{k}(\Omega)}^{2} \leq R_{k}^{2}\right\}$ is the bounded absorbing set of semigroup $S(t)$.

3) since $E_{k} \rightarrow E_{0}$ is embedded, then $E_{k}$ bounded set of compact set of $E_{0}$, so the family of semigroup operators $S(t)$ is continuous, so the equation exists

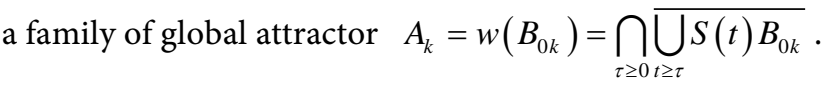

\section{Estimation of the Dimension of the Family of Global Attractor}

Firstly, we linearize the equation into a first-order variational equation and prove that the solution semigroup $S(t)$ is Fréchet differentiable on $E_{k}$, and further prove the attenuation of the volume element of the linearization problem. Finally, the upper bound of Hausdorff dimension and Fractal dimension of $A_{k}$ is estimated.

The Equations (1.1)-(1.3) is linearized

$$
\begin{gathered}
U_{t t}+M\left(\left\|\nabla^{m} u\right\|_{p}^{p}\right)(-\Delta)^{2 m} U+M^{\prime}\left(\left\|\nabla^{m} u\right\|_{p}^{p}\right)\left(\|\left.\nabla^{m} u\right|_{p} ^{p}\right)^{\prime} \nabla^{m} U(-\Delta)^{2 m} u \\
+\beta(-\Delta)^{2 m} U_{t}+\rho|u|^{\rho-1} u_{t} \cdot U+|u|^{\rho} U_{t}+(\rho+1)|u|^{\rho} U=0, \\
U(x, t)=0, x \in \partial \Omega, t>0,
\end{gathered}
$$




$$
U(x, 0)=\xi, U_{t}(x, 0)=\eta .
$$

where $(\xi, \eta) \in E_{k},\left(u, u_{t}\right)=S(t)\left(u_{0}, u_{1}\right)$ is the solution of the problem (1.1)-(1.3) with $\left(u_{0}, u_{1}\right) \in A_{k}$. Given $\left(u_{0}, u_{1}\right) \in A_{k}, S(t): E_{k} \rightarrow E_{k}$, it can be proved that for any $(\xi, \eta) \in E_{k}$, there is a unique solution $\left(U(t), U_{t}(t)\right) \in L^{\infty}\left(0,+\infty ; E_{k}\right)$ to the linearized initial-boundary value problem.

Lemma 3.1 if $S(t): E_{k} \rightarrow E_{k}$, Fréchet differential on $\eta_{0}=\left(u_{0}, u_{1}\right)$ is a linear operator $F:(\xi, \eta) \rightarrow\left(U(t), U_{t}(t)\right)$, let $t>0, R>0$, and the mapping $S(t): E_{k} \rightarrow E_{k}$ is Fréchet differentiable on $E_{k}$, where $\left(U(t), U_{t}(t)\right)$ is the solution of linearized initial-boundary value problem.

Proof.set $\eta_{0}=\left(u_{0}, u_{1}\right) \in E_{k}, \quad \bar{\eta}_{0}=\left(u_{0}+\xi, u_{1}+\eta\right) \in E_{k}$, and $\left\|\eta_{0}\right\|_{E_{k}} \leq R$, $\left\|\bar{\eta}_{0}\right\|_{E_{k}} \leq R$, make $\eta_{1}=S(t) \eta_{0}=(u, v), \quad \bar{\eta}_{1}=S(t) \bar{\eta}_{0}=(\bar{u}, \bar{v})$, In which the semigroup $S(t)$ is Lipschitz continuous on the bounded set of $E_{k}$, that is, $\left\|S(t) \eta_{0}-S(t) \bar{\eta}_{0}\right\|_{E_{k}}^{2} \leq \mathrm{e}^{C_{25} t}\|(\xi, \eta)\|_{E_{k}}^{2}$, Make $\theta=U_{t}+\varepsilon U$. so you can get it.

$$
\begin{gathered}
\theta_{t}-\varepsilon \theta+\varepsilon^{2} U+M\left(\left\|\nabla^{m} u\right\|_{p}^{p}\right)(-\Delta)^{2 m} U+M^{\prime}\left(\left\|\nabla^{m} u\right\|_{p}^{p}\right)\left(\left\|\nabla^{m} u\right\|_{p}^{p}\right)^{\prime} \nabla^{m} U(-\Delta)^{2 m} u \\
+\beta(-\Delta)^{2 m}(\theta-\varepsilon U)+\rho|u|^{\rho-1} u_{t} \cdot U+|u|^{\rho}(\theta-\varepsilon U)+(\rho+1)|u|^{\rho} U=0, \\
\theta(0)=0, \theta_{t}(0)=0 .
\end{gathered}
$$

Make $(\phi, \varphi)=(\bar{u}-u-U, \bar{v}-v-\theta)$.

$$
\left\{\begin{array}{l}
\bar{u}_{t t}+M\left(\left\|\nabla^{m} \bar{u}\right\|_{p}^{p}\right)(-\Delta)^{2 m} \bar{u}+\beta(-\Delta)^{2 m} \bar{u}_{t}+|\bar{u}|^{\rho}\left(\bar{u}_{t}+\bar{u}\right)=f(x), \\
u_{t t}+M\left(\left\|\nabla^{m} u\right\|_{p}^{p}\right)(-\Delta)^{2 m} u+\beta(-\Delta)^{2 m} u_{t}+|u|^{\rho}\left(u_{t}+u\right)=f(x), \\
U_{t t}+M\left(\left\|\nabla^{m} u\right\|_{p}^{p}\right)(-\Delta)^{2 m} U+M^{\prime}\left(\left\|\nabla^{m} u\right\|_{p}^{p}\right)\left(\left\|\nabla^{m} u\right\|_{p}^{p}\right)^{\prime} \nabla^{m} U(-\Delta)^{2 m} u \\
+\beta(-\Delta)^{2 m} U_{t}+\rho|u|^{\rho-1} u_{t} \cdot U+|u|^{\rho} U_{t}+(\rho+1)|u|^{\rho} U=0 .
\end{array}\right.
$$

Subtract these three equations to get:

$$
\begin{aligned}
& \phi_{t t}+M\left(\left\|\nabla^{m} \bar{u}\right\|_{p}^{p}\right)(-\Delta)^{2 m} \bar{u}-M\left(\left\|\nabla^{m} u\right\|_{p}^{p}\right)(-\Delta)^{2 m} u-M\left(\left\|\nabla^{m} u\right\|_{p}^{p}\right)(-\Delta)^{2 m} U \\
& -M^{\prime}\left(\left\|\nabla^{m} u\right\|_{p}^{p}\right)\left(\left\|\nabla^{m} u\right\|_{p}^{p}\right)^{\prime} \nabla^{m} U(-\Delta)^{2 m} u+\beta(-\Delta)^{2 m} \phi_{t} \\
& +|\bar{u}|^{\rho}\left(\bar{u}_{t}+\bar{u}\right)-|u|^{\rho}\left(u_{t}+u\right)-\rho|u|^{\rho-1} u_{t} \cdot U-|u|^{\rho} U_{t}-(\rho+1)|u|^{\rho} U=0 .
\end{aligned}
$$

where

$$
\begin{aligned}
H= & M\left(\left\|\nabla^{m} \bar{u}\right\|_{p}^{p}\right)(-\Delta)^{2 m} \bar{u}-M\left(\left\|\nabla^{m} u\right\|_{p}^{p}\right)(-\Delta)^{2 m} u-M\left(\left\|\nabla^{m} u\right\|_{p}^{p}\right)(-\Delta)^{2 m} U \\
& -M^{\prime}\left(\left\|\nabla^{m} u\right\|_{p}^{p}\right)\left(\left\|\nabla^{m} u\right\|_{p}^{p}\right)^{\prime} \nabla^{m} U(-\Delta)^{2 m} u+|\bar{u}|^{\rho}\left(\bar{u}_{t}+\bar{u}\right) \\
& -|u|^{\rho}\left(u_{t}+u\right)-\rho|u|^{\rho-1} u_{t} \cdot U-|u|^{\rho} U_{t}-(\rho+1)|u|^{\rho} U .
\end{aligned}
$$

Make $H=h_{1}+h_{2}$. 


$$
\begin{aligned}
h_{1}= & M\left(\left\|\nabla^{m} \bar{u}\right\|_{p}^{p}\right)(-\Delta)^{2 m} \bar{u}-M\left(\left\|\nabla^{m} u\right\|_{p}^{p}\right)(-\Delta)^{2 m} u-M\left(\left\|\nabla^{m} u\right\|_{p}^{p}\right)(-\Delta)^{2 m} U \\
& -M^{\prime}\left(\left\|\nabla^{m} u\right\|_{p}^{p}\right)\left(\left\|\nabla^{m} u\right\|_{p}^{p}\right)^{\prime} \nabla^{m} U(-\Delta)^{2 m} u \\
= & M\left(\left\|\nabla^{m} \bar{u}\right\|_{p}^{p}\right)(-\Delta)^{2 m} \bar{u}-M\left(\left\|\nabla^{m} u\right\|_{p}^{p}\right)(-\Delta)^{2 m} \bar{u}+M\left(\left\|\nabla^{m} u\right\|_{p}^{p}\right)(-\Delta)^{2 m} \phi \\
& -M^{\prime}\left(\left\|\nabla^{m} u\right\|_{p}^{p}\right)\left(\left\|\nabla^{m} u\right\|_{p}^{p}\right)^{\prime} \nabla^{m}(\bar{u}-u-\phi)(-\Delta)^{2 m} u .
\end{aligned}
$$

By the differential mean value theorem

$$
\begin{aligned}
h_{1}= & M^{\prime}\left(\left\|\nabla^{m} \bar{\zeta}\right\|_{p}^{p}\right)\left(\left\|\nabla^{m} \bar{\zeta}\right\|_{p}^{p}\right)^{\prime} \nabla^{m}(\bar{u}-u)(-\Delta)^{2 m} \bar{u} \\
& -M^{\prime}\left(\left\|\nabla^{m} u\right\|_{p}^{p}\right)\left(\left\|\nabla^{m} u\right\|_{p}^{p}\right)^{\prime} \nabla^{m}(\bar{u}-u)(-\Delta)^{2 m} u \\
& +M^{\prime}\left(\left\|\nabla^{m} u\right\|_{p}^{p}\right)\left(\left\|\nabla^{m} u\right\|_{p}^{p}\right)^{\prime} \nabla^{m} \phi(-\Delta)^{2 m} u \\
& +M\left(\left\|\nabla^{m} u\right\|_{p}^{p}\right)(-\Delta)^{2 m} \phi \\
= & M^{\prime \prime}\left(\left\|\nabla^{m} \bar{\zeta}\right\|_{p}^{p}\right)\left(\left\|\nabla^{m} \bar{\zeta}\right\|_{p}^{p}\right)^{\prime}(1-s)\left(\nabla^{m}(\bar{u}-u)\right)^{2}(-\Delta)^{2 m} \bar{u} \\
& +M^{\prime}\left(\left\|\nabla^{m} u\right\|_{p}^{p}\right)\left(\left\|\nabla^{m} u\right\|_{p}^{p}\right)^{\prime} \nabla^{m}(\bar{u}-u)(-\Delta)^{2 m}(\bar{u}-u) \\
& +M^{\prime}\left(\left\|\nabla^{m} u\right\|_{p}^{p}\right)\left(\left\|\nabla^{m} u\right\|_{p}^{p}\right)^{\prime} \nabla^{m} \phi(-\Delta)^{2 m} u+M\left(\left\|\nabla^{m} u\right\|_{p}^{p}\right)(-\Delta)^{2 m} \phi .
\end{aligned}
$$

where $\bar{\zeta}=s \nabla^{m} \bar{u}+(1-s) \nabla^{m} u, s \in(0,1), \quad \bar{\varsigma}=s \nabla^{m} \bar{\zeta}+(1-s) \nabla^{m} u, s \in(0,1)$.

Take inner product of $h_{1}$ and $(-\Delta)^{k} \phi_{t}$, there is

$$
\begin{aligned}
& \left|\left(M^{\prime \prime}\left(\left\|\nabla^{m} \bar{\zeta}\right\|_{p}^{p}\right)\left(\left\|\nabla^{m} \bar{\zeta}\right\|_{p}^{p}\right)^{\prime}(1-s)\left(\nabla^{m}(\bar{u}-u)\right)^{2}(-\Delta)^{2 m} \bar{u},(-\Delta)^{k} \phi_{t}\right)\right| \\
& \leq C_{11}\left|\int_{\Omega}\left(\nabla^{m}(\bar{u}-u)\right)^{2} \nabla^{2 m+k} \bar{u} \cdot \nabla^{2 m+k} \phi_{t} \mathrm{~d} t\right| \\
& \leq \frac{C_{12}^{2} \lambda_{1}^{-2 m-2 k}}{2 \mu_{0}}\left\|\nabla^{2 m+k}(\bar{u}-u)\right\|^{4}+\frac{\mu_{0}}{2}\left\|\nabla^{2 m+k} \phi_{t}\right\|^{2} .
\end{aligned}
$$

where $C_{11}=\left\|M^{\prime \prime}\left(\left\|\nabla^{m} \bar{\zeta}\right\|_{p}^{p}\right)\left(\left\|\nabla^{m} \bar{\zeta}\right\|_{p}^{p}\right)^{\prime}(1-s)\right\|_{\infty}, C_{12}=C_{11}\left\|\nabla^{2 m+k} \bar{u}\right\|$.

$$
\begin{aligned}
& \left|\left(M^{\prime}\left(\left\|\nabla^{m} u\right\|_{p}^{p}\right)\left(\left\|\nabla^{m} u\right\|_{p}^{p}\right)^{\prime} \nabla^{m}(\bar{u}-u)(-\Delta)^{2 m}(\bar{u}-u),(-\Delta)^{k} \phi_{t}\right)\right| \\
& \leq C_{13}\left|\int_{\Omega}\left(\nabla^{m}(\bar{u}-u)\right) \cdot \nabla^{2 m+k}(\bar{u}-u) \cdot \nabla^{2 m+k} \phi_{t} \mathrm{~d} x\right| \\
& \leq C_{13} \lambda_{1}^{-\frac{m+k}{2}}\left\|\nabla^{2 m+k}(\bar{u}-u)\right\|^{2}\left\|\nabla^{2 m+k} \phi_{t}\right\| \\
& \leq \frac{\mu_{0}}{2}\left\|\nabla^{2 m+k} \phi_{t}\right\|^{2}+\frac{C_{13}^{2} \lambda_{1}^{-m-k}}{2 \mu_{0}}\left\|\nabla^{2 m+k}(\bar{u}-u)\right\|^{4} .
\end{aligned}
$$




$$
\begin{aligned}
& \left|\left(M^{\prime \prime}\left(\left\|\nabla^{m} u\right\|_{p}^{p}\right)\left(\left\|\nabla^{m} u\right\|_{p}^{p}\right)^{\prime} \nabla^{m} \phi \cdot(-\Delta)^{2 m} u,(-\Delta)^{k} \phi_{t}\right)\right| \\
& \leq C_{14}\left|\int_{\Omega} \nabla^{m} \phi \cdot \nabla^{2 m+k} u \cdot \nabla^{2 m+k} \phi_{t} \mathrm{~d} x\right| \\
& \leq C_{14}\left\|\nabla^{m} \phi\right\|\left\|\nabla^{2 m+k} \phi_{t}\right\| \\
& \leq \frac{\mu_{0}}{2}\left\|\nabla^{2 m+k} \phi_{t}\right\|^{2}+\frac{C_{15}^{2} \lambda_{1}^{-m-k}}{\mu_{0}}\left\|\nabla^{2 m+k} \phi\right\|^{2} . \\
& \left|\left(M\left(\left\|\nabla^{m} u\right\|_{p}^{p}\right)(-\Delta)^{2 m} \phi,(-\Delta)^{k} \phi_{t}\right)\right| \geq \frac{\mu_{0}}{2} \frac{\mathrm{d}}{\mathrm{d} t}\left\|\nabla^{2 m+k} \phi\right\|^{2} .
\end{aligned}
$$

The $h_{2}=|\bar{u}|^{\rho}\left(\bar{u}_{t}+\bar{u}\right)-|u|^{\rho}\left(u_{t}+u\right)-\rho|u|^{\rho-1} u_{t} \cdot U-|u|^{\rho} U_{t}-(\rho+1)|u|^{\rho} U$.

Take inner product of $h_{2}$ and $(-\Delta)^{k} \phi_{t}$.

$$
\begin{aligned}
& \left|\left(|\bar{u}|^{\rho}\left(\bar{u}_{t}+\bar{u}\right)-|u|^{\rho}\left(u_{t}+u\right)-\rho|u|^{\rho-1} u_{t} \cdot U-|u|^{\rho} U_{t}-(\rho+1)|u|^{\rho} U,(-\Delta)^{k} \phi_{t}\right)\right| \\
& \leq C_{16}\left|\int_{\Omega} \phi_{t} \cdot(-\Delta)^{k} \phi_{t} \mathrm{~d} x\right| \leq C_{16} \lambda_{1}^{-k} \|\left.\nabla^{2 k} \phi_{t}\right|^{2} .
\end{aligned}
$$

where

$$
\begin{aligned}
\left|h_{2}\right|= & \left|\left(|\bar{u}|^{\rho} \bar{u}_{t}-|u|^{\rho} u_{t}-|u|^{\rho} U_{t}\right)+\left(|\bar{u}|^{\rho} \bar{u}-|u|^{\rho} u-\left(\rho|u|^{\rho-1} u_{t}+(\rho+1)|u|^{\rho}\right) U\right)\right| \\
\leq & C_{17}\left(|\bar{u}|^{\rho}+|u|^{\rho}\right) w_{t}-|u|^{\rho} U_{t}+C_{18}\left(|\bar{u}|^{\rho}+|u|^{\rho}\right) w_{t}-\left(\rho|u|^{\rho-1} u_{t}+(\rho+1)|u|^{\rho}\right) U \\
\leq & C_{19}\left(C_{17}\left(|\bar{u}|^{\rho}+|u|^{\rho}\right)+|u|^{\rho}\right) \phi_{t} \\
& +C_{20}\left(C_{18}\left(|\bar{u}|^{\rho}+|u|^{\rho}\right)+\left(\rho|u|^{\rho-1} u_{t}+(\rho+1)|u|^{\rho}\right)\right) \phi_{t} \\
\leq & C_{21} \phi_{t}
\end{aligned}
$$

Combined with (3.11)-(3.15), there are

$$
\begin{aligned}
& \frac{\mathrm{d}}{\mathrm{d} t}\left(\left\|\nabla^{2 k} \phi_{t}\right\|^{2}+\mu_{0}\left\|\nabla^{2 m+k} \phi\right\|^{2}\right) \\
& \leq \frac{C_{12}^{2} \lambda_{1}^{-2 m-2 k}+C_{13}^{2} \lambda_{1}^{-m-k}}{\mu_{0}}\|\bar{u}-u\|_{E_{k}}^{4}+C_{22}\left(\left\|\nabla^{2 k} \phi_{t}\right\|^{2}+\mu_{1}\left\|\nabla^{2 m+k} \phi\right\|^{2}\right) .
\end{aligned}
$$

Through the Gronwalls inequality, there is

$$
\left\|\nabla^{2 k} \phi_{t}\right\|^{2}+\mu_{0}\left\|\nabla^{2 m+k} \phi\right\|^{2} \leq C_{22} \mathrm{e}^{C_{23}}\left\|(\xi, \eta)^{\prime}\right\|_{E_{k}}^{4} .
$$

When $\left\|(\xi, \eta)^{\prime}\right\|_{E_{k}}^{4} \rightarrow 0$

$$
\frac{\|s(t) \bar{\eta}-s(t) \eta-F s(t)(\xi, \eta)\|}{\|(\xi, \eta)\|_{E_{k}}^{2}} \leq C_{24} \mathrm{e}^{C_{25} t}\|(\xi, \eta)\|_{E_{k}}^{2} \rightarrow 0 .
$$

So the lemma 3.1 is proved.

Lemma 3.2 Under the assumption and condition of lemma 3.1, the family of global attractor $A_{k}$ of initial-boundary value problem (1.1)-(1.3) has Hausdoff 
dimension and Fractal dimension, and $d_{H}\left(A_{k}\right) \leq \frac{1}{7} n, d_{F}\left(A_{k}\right) \leq \frac{8}{7} n$.

Proof. Make $\phi=R_{\varepsilon} \varphi=(u, v)^{\prime}, \varphi=\left(u, u_{t}\right)^{\prime}, \quad v=u_{t}+\varepsilon u$,

$R_{\varepsilon}:\left\{u, u_{t}\right\} \rightarrow\left\{u, u_{t}+\varepsilon u\right\}$ is an isomorphic mapping, let $A_{i}(i=1,2, \cdots, 2 m)$ be the global attractor of $\{S(t)\}$, and $A_{\varepsilon i}$ is the global attractor of $\left\{S_{\varepsilon}(t)\right\}$, and they have the same dimension, from lemma 3.1, we can know that $S(t): E_{k} \rightarrow E_{k}$ is Fréchet differentiable. The linearized first-order variational Equation (3.1) can be rewritten as

$$
\begin{gathered}
P_{t}+\Lambda(\Psi) P=0 . \\
P_{t}=F_{t}(\Psi) .
\end{gathered}
$$

where

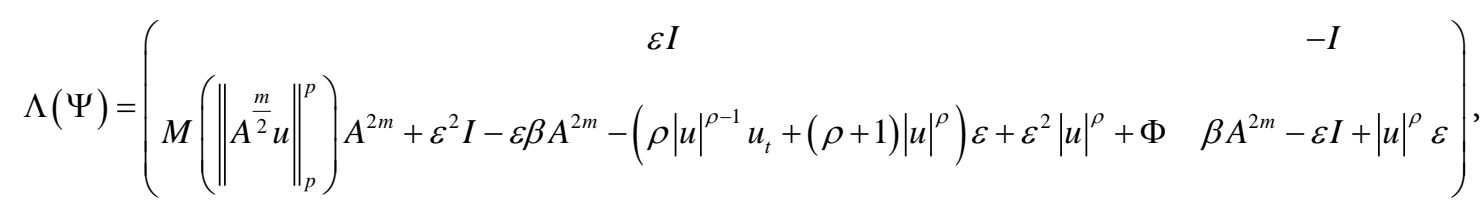

$I$ is an identity operator. $-\Delta=A, \quad P=(U, \theta)^{\prime} \in E_{k}$. Make $\Psi=R_{\varepsilon} \varphi=(U, V)$, $\varphi=\left(U, U_{t}\right), \quad \theta=U_{t}+\varepsilon U \quad R_{\varepsilon}:\left\{u, u_{t}\right\} \rightarrow\left\{u, u_{t}+\varepsilon u\right\}$ is an isomorphic mapping. For a fixed $\left(u_{0}, v_{0}\right) \in E_{k}$, let $r_{1}, r_{2}, \cdots, r_{n}$ be $n$ element of $E_{k}$, let $U_{1}(t), U_{2}(t), \cdots, U_{n}(t)$ be $n$ solutions of the linear Equation (3.19), its initial value is $U_{1}(0)=r_{1}, U_{2}(0)=r_{2}, \cdots, U_{n}(0)=r_{n}$.

so

$$
\begin{aligned}
& \frac{\mathrm{d}}{\mathrm{d} t}\left\|U_{1}(t) \Lambda U_{2}(t) \Lambda \cdots \Lambda U_{n}(t)\right\|_{\Lambda E_{k}}^{2} \\
& -2 \operatorname{tr} F_{t}\left(\Psi(\tau) \cdot Q_{n}(\tau)\right)\left\|U_{1}(t) \Lambda U_{2}(t) \Lambda \cdots \Lambda U_{n}(t)\right\|_{\Lambda E_{k}}^{2}=0 .
\end{aligned}
$$

Further, by the same GronwalPs inequality, available:

$$
\begin{aligned}
& \left\|U_{1}(t) \Lambda U_{2}(t) \Lambda \cdots \Lambda U_{n}(t)\right\|_{\Lambda E_{k}}^{2} \\
& =\left\|r_{1} \Lambda r_{2} \Lambda \cdots \Lambda r_{n}\right\|_{\Lambda E_{k}} \exp \left(\int_{0}^{t} \operatorname{tr} F_{t}\left(\Psi(\tau) \cdot Q_{n}(\tau)\right) \mathrm{d} \tau\right) .
\end{aligned}
$$

where $\Lambda$ stands for outer product and tr stands for trace. $Q_{n}(\tau)$ is an orthogonal projection from space $E_{k}$ to $\operatorname{span}\left\{U_{1}(t), U_{2}(t), \cdots, U_{n}(t)\right\}$.

Given a certain moment $\tau$,

set $w_{j}(\tau)=\left(\xi_{j}(\tau), \eta_{j}(\tau)\right)^{\prime}, j=1,2, \cdots, n$ is $\operatorname{span}\left\{U_{1}(t), U_{2}(t), \cdots U_{n}(t)\right\}$ orthonormal basis.

We define the inner product in $E_{k}$ is

$$
((\xi, \eta),(\bar{\xi}, \bar{\eta}))=\left(\left(\nabla^{2 m+k} \xi, \nabla^{2 m+k} \bar{\xi}\right)+\left(\nabla^{k} \eta, \nabla^{k} \bar{\eta}\right)\right) .
$$

To sum up, it is available

$$
\begin{aligned}
\operatorname{tr} F^{\prime}\left(\Psi(\tau) \cdot Q_{n}(\tau)\right) & =\sum_{j=1}^{n}\left(F^{\prime}(\Psi(\tau)) \cdot Q_{n}(\tau) w_{j}(\tau), w_{j}(\tau)\right)_{E_{k}} \\
& =\sum_{j=1}^{n}\left(F^{\prime}(\Psi(\tau)) w_{j}(\tau) \cdot w_{j}(\tau)\right)_{E_{k}} .
\end{aligned}
$$




$$
\begin{aligned}
\text { where } & \left(F^{\prime}(\Psi(\tau)) w_{j}(\tau) \cdot w_{j}(\tau)\right)_{E_{k}}=-\left(\Lambda(\Psi) w_{j}, w_{j}\right) . \\
= & -\varepsilon\left\|\nabla^{2 m+k} \xi_{j}\right\|^{2}+\left(\nabla^{2 m+k} \xi_{j}, \nabla^{2 m+k} \eta_{j}\right)-M\left(\left\|A^{\frac{m}{2}} u\right\|_{p} \|^{p}\left(\nabla^{2 m+k} \xi_{j}, \nabla^{2 m+k} \eta_{j}\right)\right. \\
& -\varepsilon^{2}\left(\nabla^{k} \xi_{j}, \nabla^{k} \eta_{j}\right)+\beta \varepsilon\left(\nabla^{2 m+k} \xi_{j}, \nabla^{2 m+k} \eta_{j}\right) \\
& +\varepsilon\left(\rho|u|^{\rho-1} u_{t}+(\rho+1)|u|^{\rho}\right)\left|u_{t}\right|^{\rho-1}\left(\nabla^{k} \xi_{j}, \nabla^{k} \eta_{j}\right)-\Phi\left(\nabla^{k} \xi_{j}, \nabla^{k} \eta_{j}\right) \\
& -\beta\left\|\nabla^{2 m+k} \eta_{j}\right\|^{2}+\varepsilon\left\|\nabla^{k} \eta_{j}\right\|^{2}-\varepsilon^{2}|u|^{\rho}\left(\nabla^{k} \xi_{j}, \nabla^{k} \eta_{j}\right)-\varepsilon|u|^{\rho}\left\|\nabla^{k} \eta_{j}\right\|^{2} \\
\leq- & \varepsilon\left\|\nabla^{2 m+k} \xi_{j}\right\|^{2}+\frac{\mu_{0}-\beta \varepsilon-1}{2}\left\|\nabla^{2 m+k} \xi_{j}\right\|^{2}+\frac{\mu_{0}-\beta \varepsilon-1}{2}\left\|\nabla^{2 m+k} \eta_{j}\right\|^{2}+\frac{\varepsilon}{2}\left\|\nabla^{k} \xi_{j}\right\|^{2} \\
+ & \frac{\Phi+\varepsilon^{2}|u|^{\rho}-\varepsilon\left(\rho|u|^{\rho-1} u_{t}+(\rho+1)|u|^{\rho}\right)}{2 \lambda_{1}^{2 m}}\left\|\nabla^{2 m+k} \xi_{j}\right\|^{2} \\
+ & \frac{\Phi+\varepsilon^{2}|u|^{\rho}-\varepsilon\left(\rho|u|^{\rho-1} u_{t}+(\rho+1)|u|^{\rho}\right)+3 \varepsilon+2 \varepsilon|u|^{\rho}-2 \beta \lambda_{1}^{2 m}}{2}\left\|\nabla^{k} \eta_{j}\right\|^{2} \\
\leq & -C_{26}\left(\left\|\nabla^{2 m+k} \xi_{j}\right\|^{2}+\left\|\nabla^{k} \eta_{j}\right\|^{2}\right)+\frac{\varepsilon}{2}\left\|\nabla^{k} \xi_{j}\right\|^{2} .
\end{aligned}
$$

where

$$
\begin{aligned}
C_{26}= & \min \left\{-\left(\frac{\mu_{0}-\beta \varepsilon-1}{2}-\varepsilon+\frac{\Phi+\varepsilon^{2}|u|^{\rho}-\varepsilon\left(\rho|u|^{\rho-1} u_{t}+(\rho+1)|u|^{\rho}\right)}{2 \lambda_{1}^{2 m}}\right),\right. \\
& \left.-\left(\frac{\mu_{0}-\beta \varepsilon-1}{2} \lambda_{1}^{2 m}+\frac{\Phi+\varepsilon^{2}|u|^{\rho}-\varepsilon\left(\rho|u|^{\rho-1} u_{t}+(\rho+1)|u|^{\rho}\right)+3 \varepsilon+2 \varepsilon|u|^{\rho}-2 \beta \lambda_{1}^{2 m}}{2}\right)\right\}
\end{aligned}
$$

Above all there is

$$
\left(F_{t}(\Psi(\tau)) w_{j}(\tau), w_{j}(\tau)\right) \leq-C_{26}\left(\left\|\nabla^{2 m+k} \xi_{j}\right\|^{2}+\left\|\nabla^{k} \eta_{j}\right\|^{2}\right)+\frac{\varepsilon}{2}\left\|\nabla^{k} \xi_{j}\right\|^{2} .
$$

Because $w_{j}(\tau)=\left(\xi_{j}(\tau), \eta_{j}(\tau)\right)^{\mathrm{T}}, j=1,2, \cdots, n$ is $\operatorname{span}\left\{U_{1}(t), U_{2}(t), \cdots, U_{n}(t)\right\}$ orthonormal basis. Therefore

$$
\begin{gathered}
\left\|\nabla^{2 m+k} \xi_{j}\right\|^{2}+\left\|\nabla^{k} \eta_{j}\right\|^{2}=1 . \\
\sum_{j=1}^{n}\left(F^{\prime}(\Psi(\tau)) w_{j}(\tau), w_{j}(\tau)\right)_{E_{k}} \leq-n C_{26}+\frac{\varepsilon}{2} \sum_{j=1}^{n}\left\|\nabla^{k} \xi_{j}\right\|^{2} .
\end{gathered}
$$

Almost all the $t$.

$$
\sum_{j=1}^{n}\left\|\nabla^{k} \xi_{j}\right\|^{2} \leq \sum_{j=1}^{n} \lambda_{j}^{s-1}
$$

The $s-1=-2 m$ and $s \in[0,1], \quad \lambda_{j}$ is $A^{2 m}$ characteristic value, and $\lambda_{1}<\lambda_{2}<\cdots<\lambda_{n}$, so there is

$$
\operatorname{TrF}^{\prime}\left(\Psi(\tau) \cdot Q_{n}(\tau)\right) \leq-n C_{26}+\varepsilon \sum_{j=1}^{n} \lambda_{j}^{s-1} .
$$

Set 


$$
q_{n}(t)=\sup _{\Psi_{0} \in B_{0 k}} \sup _{\eta_{j} \in E_{k}}\left(\frac{1}{t} \int_{0}^{t} \operatorname{Tr} F^{\prime}\left(s(t) \Psi_{0}\right) Q_{n}(\tau) \mathrm{d} \tau\right)
$$

and

$$
q_{n}=\lim _{t \rightarrow \infty} q_{n}(t)
$$

Therefore,

$$
q_{n} \leq-n C_{26}+\varepsilon \sum_{j=1}^{n} \lambda_{j}^{s-1}
$$

Therefore, $B_{0 k}$ Lyapunov index $\kappa_{1}, \kappa_{2}, \cdots, \kappa_{n}(n>1)$ is uniformly bounded, and

$$
\kappa_{1}+\kappa_{2}+\cdots+\kappa_{n} \leq-n C_{26}+\varepsilon \sum_{j=1}^{n} \lambda_{j}^{s-1}
$$

make

$$
\begin{aligned}
& \left(q_{j}\right)_{+} \leq-n C_{26}+\varepsilon \sum_{j=1}^{n} \lambda_{j}^{s-1} \leq \varepsilon \sum_{j=1}^{n} \lambda_{j}^{s-1} \leq \frac{n C_{26}}{8} . \\
& q_{n} \leq-n C_{26}\left(1-\frac{\varepsilon}{n C_{26}} \sum_{j=1}^{n} \lambda_{j}^{s-1}\right) \leq-\frac{7}{8} n C_{26} .
\end{aligned}
$$

Further,

$$
\max _{1 \leq j \leq n} \frac{\left(q_{j}\right)_{+}}{\left|q_{n}\right|} \leq \frac{1}{7}
$$

From this we can get $d_{H}\left(A_{k}\right) \leq \frac{1}{7} n, d_{F}\left(A_{k}\right) \leq \frac{8}{7} n$, Then the Hausdorff dimension and Fractal dimension of the family of global attractor $A_{k}$ are finite.

\section{Conflicts of Interest}

The authors declare no conflicts of interest regarding the publication of this paper.

\section{References}

[1] Cavalcanti, M.M., Cavalcanti, V.N.D., Filho, J.S.P. and Soriano, J.A. (1998) Existence and Exponential Decay for a Kirchhoff-Carrier Model with Viscosity. Journal of Mathematical Analysis and Applications, 226, 40-60.

https://doi.org/10.1006/jmaa.1998.6057

[2] Ono, K. (1997) Global Existence, Decay, and Blow up of Solutions for Some Mildly Degenerate Nonlinear Kirchhoff Strings. Journal of Differential Equations, 137, 273-301. https://doi.org/10.1006/jdeq.1997.3263

[3] Ono, K. (1997) On Global Existence, Asymptotic Stability and Blowing up of Solutions for Some Degenerate Non-Linear Wave Equtions of Kirchhoff Type with a Strong Dissipation. Mathematical Methods in the Applied Sciences, 20, 151-177. https://doi.org/10.1002/(SICI)1099-1476(19970125)20:2<151::AID-MMA851>3.0.C $\underline{\mathrm{O} ; 2-0}$

[4] Yang, Z.J. (2007) Longtime Behavior of the Kirchhoff Type Equation with Strong 
Damping on $R^{N}$. Journal of Differential Equations, 242, 269-286.

[5] Yang, Z.J., Ding, P.Y. and Liu, Z.M. (2014) Global Attractor for the Kirchhoff Type Equations with Strong Nonlinear Damping and Supercritical Nonlinearity. Applied Mathematics Letters, 33, 12-17. https://doi.org/10.1016/j.aml.2014.02.014

[6] Yang, Z.J., Wang, Y.Q. (2010) Global Attractor for the Kirchhoff Type Equation with a Strong Dissipation. Journal of Differential Equations, 249, 3258-3278. https://doi.org/10.1016/j.jde.2010.09.024

[7] Yang, Z. and Li, X. (2011) Finite Dimensional Attractors for the Kirchhoff Equation with a Strong Dissipation. Journal of Mathematical Analysis and Applications, 375, 579-593. https://doi.org/10.1016/j.jmaa.2010.09.051

[8] Lin, G.G. (1973) Several Types of the Kirchhoff Equation of Dynamics Characteristic. Theoretical Population Biology, 4, 331-356.

[9] Tokio, M. and Ryo, I. (1996) On Global Solutions and Energy Decay for the Wave Equations of Kirchhoff Type with Nonlinear Damping Terms. Journal of Mathematical Analysis and Applications, 204, 729-753.

https://doi.org/10.1006/jmaa.1996.0464

[10] Li, F.C. (2004) Global Existence and Blow-up of Solutions for a Higher-Order Kirchhoff-Type Equations with Nonlinear Dissipation. Applied Mathematics letters, 17, 1409-1414. https://doi.org/10.1016/j.am1.2003.07.014

[11] Lin, G.G. (2011) Nonlinear Evolution Equations. Kunming: Yunnan University Press.

[12] Gao, Y.L., Sun, Y.T. and Lin, G.G. (2016) The Global Attractors and Their Hausdorff and Fractal Dimensions Estimation for the Higer-Order Nonlinear Kirchhoff-Type Equation with Strong Lineae Damping. International Journal of Modern Nonlinear Theory and Application, 5, 185-202. https://doi.org/10.4236/ijmnta.2016.54018

[13] Chen, L., Wang, W. and Lin, G.G. (2016) The Global Attractors and the Hausdorff and Fractal Demensions Estimation for the Higher-Order Nonlinear Kirchhoff-Type Equation. Journal of Advances in Mathematics, 12, 6608-6621. https://doi.org/10.24297/jam.v12i9.133

[14] Ma, Q.Z., Sun, C.Y. and Zhong, C.K. (2007) The Existence of Strong Global Attractors for Nonlinear Beam Equations. Journal of Mathematical Physics, 27A, 941-948. 